\title{
Silicon Micromachined Hollow Microneedles for Transdermal Liquid Transport
}

\author{
Han J. G. E. Gardeniers, Regina Luttge, Erwin J. W. Berenschot, Meint J. de Boer, Shuki Y. Yeshurun, Meir Hefetz, \\ Ronny van't Oever, and Albert van den Berg
}

\begin{abstract}
This paper presents a novel process for the fabrication of out-of-plane hollow micro needles in silicon. The fabrication method consists of a sequence of deep-reactive ion etching (DRIE), anisotropic wet etching and conformal thin film deposition, and allows needle shapes with different, lithographydefined tip curvature. In this study, the length of the needles varied between 150 and 350 micrometers. The widest dimension of the needle at its base was $250 \mu \mathrm{m}$. Preliminary application tests of the needle arrays show that they are robust and permit skin penetration without breakage. Transdermal water loss measurements before and after microneedle skin penetration are reported. Drug delivery is increased approximately by a factor of 750 in microneedle patch applications with respect to diffusion alone. The feasibility of using the microneedle array as a blood sampler on a capillary electrophoresis chip is demonstrated.
\end{abstract}

[999]

Index Terms-Deep-reactive ion etching (DRIE), diagnostics, drug delivery, microneedle, point-of-care, transdermal.

\section{INTRODUCTION}

$\mathbf{N}$ OWADAYS, typical routes for drug delivery are either through hypodermic needles, via (iontophoretic) patches or by oral or respiratory administration, while diagnostic sampling in most cases requires extraction of blood through a hypodermic syringe needle, followed by analysis of blood components in a specialized laboratory environment. These methods all have some disadvantages, depending on the particular drug that is to be administered, or the analysis that is to be performed. For example, in some cases the methods are too slow, not effective, or wasteful with respect to the volumes of blood or other substances that are consumed, or receive insufficient patient's compliance, because of pain and skin irritation or skin damage.

The introduction of MEMS offers exciting opportunities to advance the minimally invasive medical field. In addition, miniaturization of analysis methods enables the development of versatile portable equipment for "Point-of-care" monitoring

Manuscript received January 24, 2003; revised August 7, 2003. This work was supported by NanoPass Ltd., Israel. Subject Editor K. D. Wise.

H. J. G. E. Gardeniers was with Micronit Microfluidics BV, Enschede, The Netherlands. He is now with the MESA + Research Institute, University of Twente, 7500 AE Enschede, The Netherlands (e-mail: j.g.e.gardeniers@utwente.nl).

R. Luttge, E. J. W. Berenschot, M. J. de Boer, and A. van den Berg are with the MESA + Research Institute, University of Twente, 7500 AE Enschede, The Netherlands.

S. Y. Yeshurun and M. Hefetz are with NanoPass Technologies, Ltd., Haifa 31043, Israel.

R. van't Oever is with Micronit Microfluidics BV, 7500 AE Enschede, The Netherlands.

Digital Object Identifier 10.1109/JMEMS.2003.820293

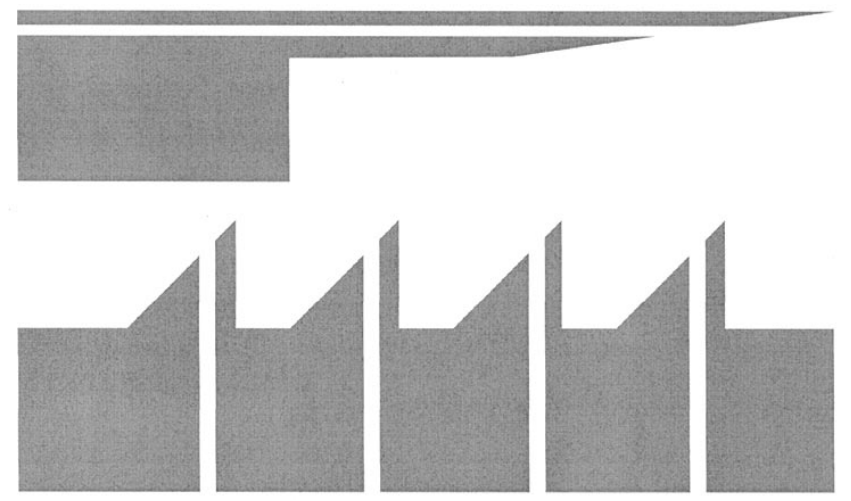

Fig. 1. Two types of microneedles. Top: in-plane design. Bottom: out-of-plane design. Drawings are cross sections along the length of the needles.

and treatment of patients. Ultimately, drug-on-demand possibilities, using micro system technology to integrate monitoring and dispensing components in an intelligent feedback system that is so small that it can be carried on the body of the patient without obstructing his movement, may become feasible.

In this paper we focus on the micromachining of an array of hollow microneedles for transdermal liquid transfer. Such hollow needles can be used for body fluid sampling (e.g., blood or interstitial fluid) or drug delivery. Drug infusion applications require that the drug is injected into the viable epidermis, which forms a cell layer extending to a depth of 60-130 $\mu \mathrm{m}$ below the stratum corneum (the 10-20 $\mu \mathrm{m}$ thick outer layer of the skin, which forms the main barrier for transport of drugs across the skin). For blood withdrawal, a deeper penetration is generally required, to ca. $1 \mathrm{~mm}$ below the surface of the skin, into the 1-2 mm thick dermis that contains the blood vessels and the nerve ends.

The basic idea behind the microneedle approach is that due to the small size of the needles, tissue damage will be limited and pain sensation can be reduced [1] or even completely avoided. To obtain the necessary fluid flow (generally of the order of below $1 \mu \mathrm{l}$ per hour up to ca. $100 \mu \mathrm{l}$ per hour) for acceptable pumping pressures, a large number of microneedles on the same device area can be used.

Several approaches to the micromachining of this type of device are known, and roughly these can be divided in in-plane and out-of-plane designs, the plane in this case being the surface of e.g., a silicon wafer. Fig. 1 shows schematic drawings of both these needle types. The in-plane version is the most convenient to fabricate with state-of-the-art planar technology, comprising surface micromachining and different techniques of (sil- 
icon) etching, and creates a good degree of flexibility with respect to different needle designs. Illustrative examples are the sophisticated hollow neural probes of Wise et al., fabricated by anisotropic wet etching of a silicon substrate combined with deep diffused boron etch stops and having CMOS electronic circuitry integrated on the chip [2], [3], the microneedles manufactured by anisotropic wet etching of a silicon substrate, with surface micromachined polysilicon-based fluidic channels on them, by Pisano's group [4], [5], which were later refined by the use of silicon-on-insulator substrates and isotropic etching [6], the hollow micromachined needle arrays by Brazzle et al. [7], [8], and the surface micromachined polysilicon microneedles with permeable polysilicon on one side which serves as a microdialysis membrane [9], [10].

The density of needles that can be obtained with the in-plane method is limited, since only one row of needles can be made per chip. Modern analytical equipment for blood analysis requires at least $5 \mu \mathrm{l}$ and more typically $50 \mu \mathrm{l}$ of blood for a reliable analysis. Therefore, for blood withdrawal, with the typical microneedle inner diameters of 10-100 $\mu \mathrm{m}$ reported in literature, a higher needle density may be desirable in order to achieve sufficient liquid flow. Furthermore, if needle diameters are made smaller in order to limit pain sensations, a larger number of parallel microneedles may give the necessary redundancy in case some of the needles for whatever reason get blocked during operation. Although it is possible to achieve high needle densities by hybrid integration of in-plane needle arrays [11], from a technological point of view the out-of-plane version provides an easier route to obtain a large density of needles per chip, while the fact that the needles in this case are backed with a few $\mathrm{mm}^{2}$ area flat plate with a thickness of less than $1 \mathrm{~mm}$ makes this configuration suitable for integration in a patch to be worn on a patient's body.

Published early examples of out-of-plane micromachined microneedles for transdermal applications [12]-[14] have as a disadvantage that their flat hollow tips tend to punch the skin, with the risk that the punched material may at least partially obstruct liquid flow through the needle. Therefore it is preferred that the flow channel through the needle is positioned off-center from the needle tip. Very promising results were obtained by Stoeber and Liepmann [15], who used directional reactive ion etching (RIE) to define a narrow flow channel through a silicon wafer and thin film protection of this channel followed by isotropic etching from the other side of the wafer to fabricate such a needle. A totally different and equally promising approach was followed by Griss and Stemme, who, using the special etching properties of RIE combined with passivation steps in-between the etching cycles, fabricated out-of-plane microneedles with openings on the side, by which clogging due to punching should be avoided [16].

In this paper we present a fabrication procedure, in which both anisotropic wet etching and directional (deep) reactive ion etching processes are combined, to achieve out-of-plane microneedle structures in array format [17] (the process and structure of the needles are patent pending [18]). The needle design anticipates a good mechanical strength and adequate skin penetration properties, while the needles contain flow channels positioned off-center of the needle tips. Needles with different and

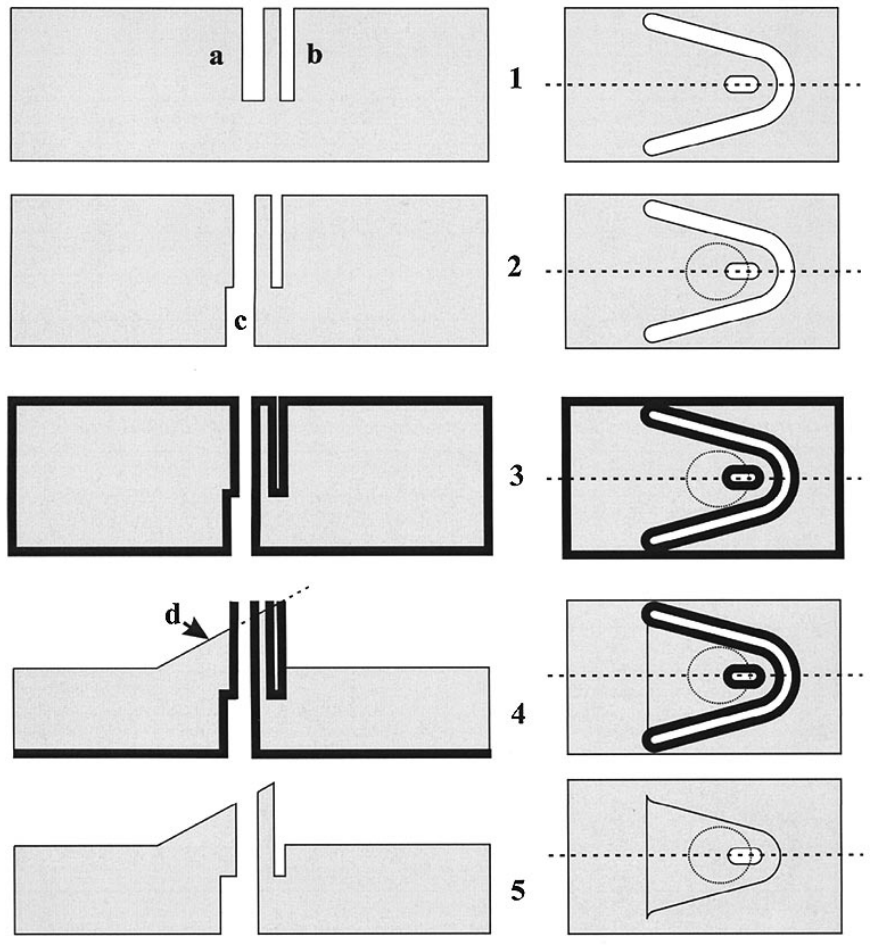

Fig. 2. Microneedle fabrication sequence. The drawings on the left give a cross section of the structure after each processing step, along the dotted line in the top view drawings that are shown on the right. The thick black line represents a silicon nitride coating that is used as a protection layer during $\mathrm{KOH}$ etching. Refer to text for details.

well-defined tip shapes were fabricated and are discussed below. The feasibility of the needles for transdermal water loss, drug delivery and diagnostics are presented.

\section{FABRICATION PROCESS}

Fig. 2 describes the fabrication process for the out-of-plane needle design. The method builds on the directional deep RIE work at cryogenic temperatures that was previously developed by us to fabricate buried microchannels in silicon [19] and anisotropic wet etching of silicon as performed by Albrecht et al. to fabricate AFM tips [20], and requires only two lithographic masks. More details about the possibilities of cryogenic RIE and the procedures that are used to achieve deep structures in a silicon substrate, were recently published in this journal by us [21].

Essential features of the design are that the location of the flow channel opening does not coincide with the needle tip and in our case can be positioned freely. This flow channel is etched as follows: The fabrication process starts with a RIE step using $\mathrm{SF}_{6}$ plasma chemistry in an Oxford Plasmalab 100 machine (typical etching rate $1.5 \mu \mathrm{m} / \mathrm{min}$ ) by which in a silicon $\{100\}$ substrate a flow channel (see Fig. 2a) is etched. This flow channel through the needle does not extend to the other side of the substrate, but is etched only as deep as is necessary to achieve the desired needle length. The length is defined by the depth of slot Fig. 2b, which is etched in the same process as the flow channel Fig. $2 \mathbf{a}$.

Next, a channel Fig. 2c that connects the flow channel through the needle to the backside of the wafer is etched by RIE with 
the same parameters as in the RIE step described above. In this step, it is possible to include a fluidic channel structure on the backside of the substrate, e.g., for sample treatment or characterization, or drug storage purposes. Furthermore, positioning of channel Fig. 2c, which can have a much wider cross-section than the flow channel through the needle, is not critical, and other etching steps may be used instead of the RIE used in this study. The requirements are that the two channels connect, and that the channel from the backside does not connect with the slot.

Subsequently the inner surfaces of the holes and the slot are coated with a conformal layer that is resistant against $\mathrm{KOH}$. In our case we used low-pressure chemical vapor deposition (LPCVD) of a low-stress (ca. $300 \mathrm{MPa}$ [22]) silicon-rich silicon nitride layer with a thickness of $500 \mathrm{~nm}$. Conditions for this deposition step were: gas flows $70 \mathrm{sccm} \mathrm{SiCl}_{2} \mathrm{H}_{2}$ and 18 sccm $\mathrm{NH}_{3}$, temperature $850^{\circ} \mathrm{C}$, pressure 200 mTorr.

After removal of the protective layer at the top surface of the wafer with a directional $\mathrm{SF}_{6}$-plasma RIE process [19], in Fig. 2, step 4 anisotropic wet etching is performed, which leaves a structure bound by a slow-etching $\{111\}$ plane on one side, indicated by Fig. 2d. A similar method was used by Albrecht et al. to fabricate AFM tips [20]. The coated slot sidewalls prevent etching from the sides. Finally, the protective silicon nitride layer is stripped in a 50\% Hydrogen Fluoride (HF) solution. This etchant removes the silicon nitride layer at a rate of ca. $4 \mathrm{~nm} / \mathrm{min}$, but since the selectivity of silicon nitride etching with respect to single-crystalline silicon is extremely high, the exact etching time is not critical.

Fig. 3(a) shows a scanning electron microscope (SEM) photograph of the resulting structure after in Fig. 2, step 4. Fig. 3(b) gives a schematic drawing of a similar structure as shown in Fig. 3(a), also after Fig. 2, step 4. It can be seen that the needle boundaries are determined by the slow-etching $\mathrm{Si}\{111\}$ plane and the nonetching silicon nitride walls of the slot. The needle tip shape and tip position are determined by the alignment of the crystallographic directions in the silicon with the design of the slot. The advantage of this method over that of Stoeber et $a l$. , who etched the connecting hole through the wafer, starting from the backside of the substrate up to the surface at which the needle tip is defined later, is that the essential lithographic definition of respective hole (flow channel through needle) and needle tip positions is done on the same side of the wafer. Therefore in our case back-to-front alignment is less critical, and the shape of the flow channel at the position where it really matters, i.e., at the needle surface, is precisely defined.

\section{FABRICATION RESULTS}

Fig. 4 shows a typical result of a $350 \mu \mathrm{m}$ high microneedle with a triangular tip shape, a base of $250 \mu \mathrm{m}$, and a maximum hole width of $70 \mu \mathrm{m}$. The center of the elliptical flow channel in the needle is positioned ca. $40 \mu \mathrm{m}$ from the tip of the needle. It can be seen that the vertical sidewall of the needle has a rough surface, which is caused by photolithographic mask edge imperfections in combination with the characteristics of the applied RIE process. It is unknown whether this roughness will affect the performance of the needles for skin penetration.

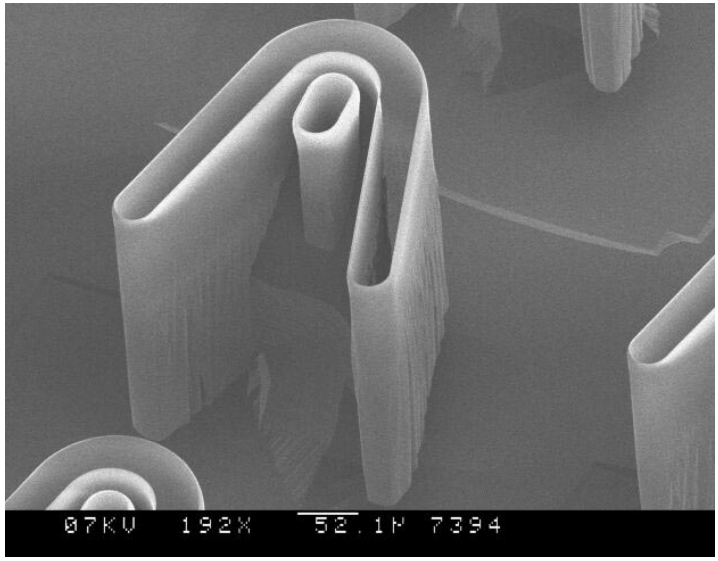

(a)

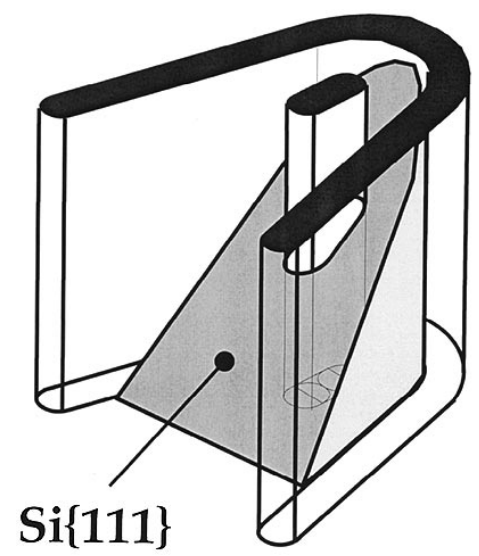

(b)

Fig. 3. (a) The slot and flow channel, bound by silicon nitride walls that are resistant against $\mathrm{KOH}$ etching, after step 4 of the process sequence in Fig. 2 b Schematic drawing of slot walls, flow channel walls, and remaining needle. The dark gray area is the $\mathrm{Si}\{111\}$ surface, the light gray area is the side face of the needle as it is defined by the walls of the silicon nitride coated slots.

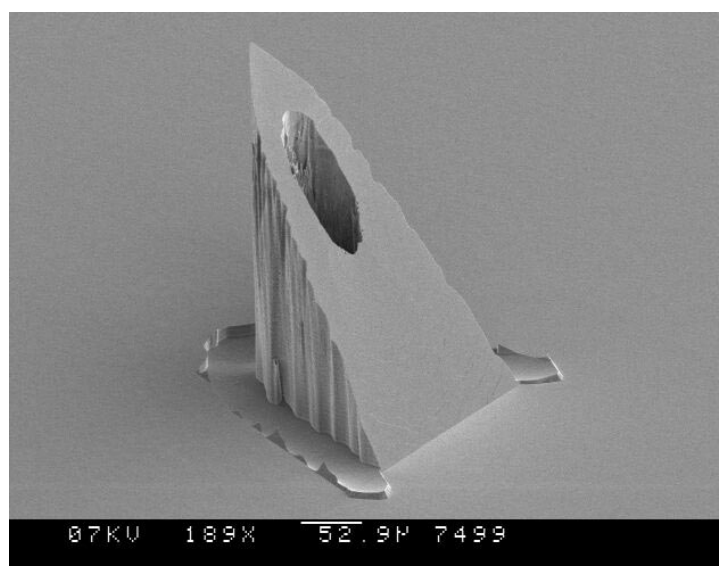

Fig. 4. SEM picture of a $350 \mu \mathrm{m}$ high microneedle, with a base of $250 \mu \mathrm{m}$ (measured in widest direction). The elliptical flow channel is $70 \mu \mathrm{m}$ in its widest direction.

The needle length as it was fabricated here is sufficient for most drug infusion applications. The fabrication method presented here also allows needles with the appropriate length for blood withdrawal, however in our case the length was limited by the thickness of the $100 \mathrm{~mm}$ diameter silicon wafers, which was approximately $530 \mu \mathrm{m}$. 


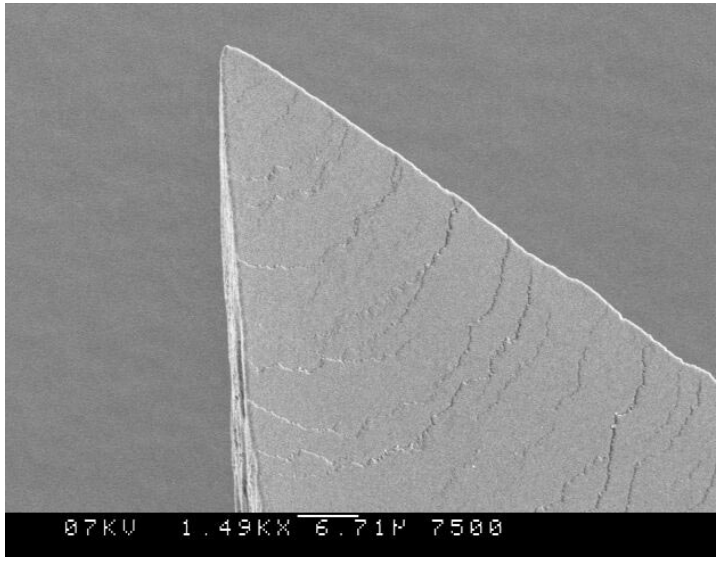

Fig. 5. Close-up of the tip of the needle of Fig. 4. Note the steps present on the surface.

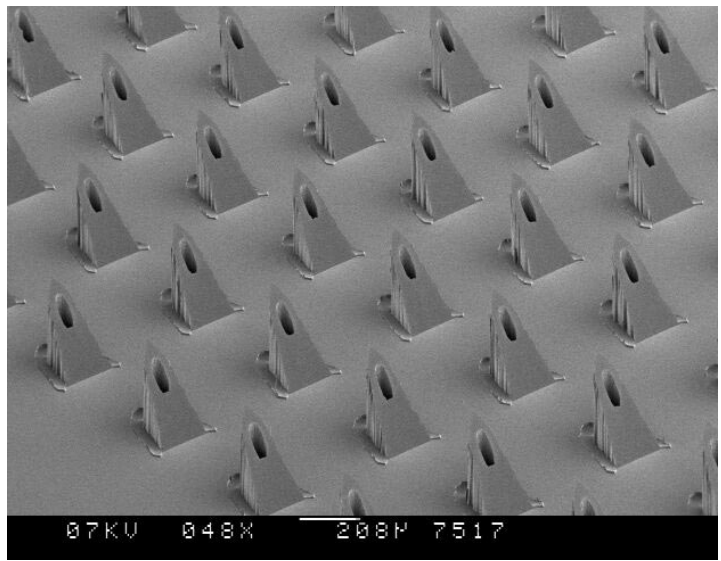

Fig. 6. Array of needles of Fig. 4, with a pitch of $555 \mu \mathrm{m}$.

Fig. 5 shows a close-up of the sloped sidewall of the needle, which is formed by a silicon surface that is oriented along the $\{111\}$ plane. The picture shows a number of very low steps, originating from the tip of the needle that was originally bound by the protective layer of the slot. These steps are a typical feature of the etching process on an atomically flat crystal plane being in contact with a nonetching material [23], and are also observed on the pyramidal hillocks that frequently form on silicon $\{001\}$ surfaces during etching in $\mathrm{KOH}$ solutions [24]. Due to these "step trains," the slope of the needle surface is somewhat less than that of a perfectly oriented $\{111\}$ plane. This has less to do with the fact that the top of the needle has been exposed longer to the etchant than the base, but is due to the nucleation of etching steps at the lines where the silicon $\{111\}$ surface meets the silicon nitride walls. This effect was studied in detail and explained in a recent paper by van Veenendaal $e t$ al. [25].

The presented fabrication method allows a high needle density, see Fig. 6, with excellent uniformity across the wafer surface. Other needle tip designs can easily be achieved by changing the design of the slot, see Fig. 7 which shows a microneedle with a round tip shape.

As was learned from discussions with several European silicon micromachining foundries, the procedures used for the fabrication of the needles can be developed into a large-volume

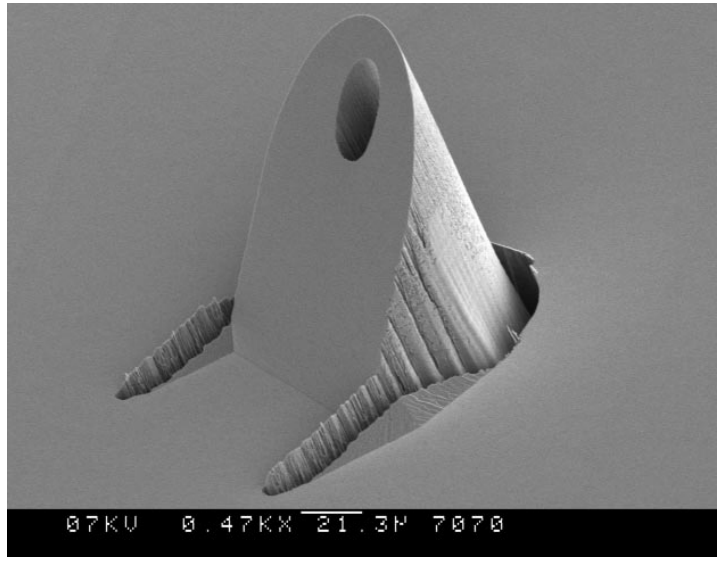

Fig. 7. SEM picture of a microneedle with a tip with large radius of curvature.

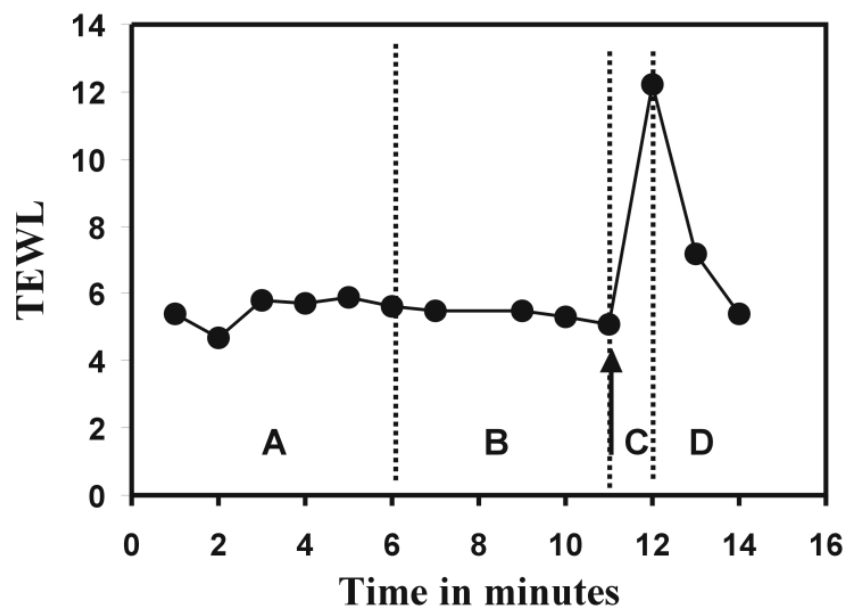

Fig. 8. Measurement of transdermal water loss with and without a microneedle chip with a 6 by 6 array of $350 \mu \mathrm{m}$ height. A: without chip; B: chip with backside to skin; C: with needle array inserted (at the time indicated by the arrow); D: with needle chip removed.

production process. Smaller volume production runs have already been performed (Micronit Microfluidics B.V.).

\section{Performance Tests}

The effectiveness of the microneedle array for transdermal application was tested by measuring the transdermal water loss (TEWL) on human skin. Transdermal water loss rates can be used to monitor changes in the barrier function of the stratum corneum. The test was conducted using standard CORTEX DermaLab equipment [26] which has a probe that is placed on the skin; the probe contains two humidity sensors that measure the water vapor pressure gradient in the direction perpendicular to the skin, which gradient is a measure for the TEWL [27]. Environmental conditions during the tests were: relative humidity (RH) $29.1 \%$, temperature $22.0^{\circ} \mathrm{C}$.

Fig. 8 shows a typical result. The test procedure was as follows: first the TEWL (in $\mathrm{g} \mathrm{hr}^{-1} \mathrm{~m}^{-2}$ ) was measured without chip (see zone A in Fig. 8) at intervals of $1 \mathrm{~min}$. The 1-min interval is necessary to ensure that steady-state conditions are obtained inside the probe, which in most cases take 30-45 s [28]. Next, a reference test was performed, in which the microneedle 


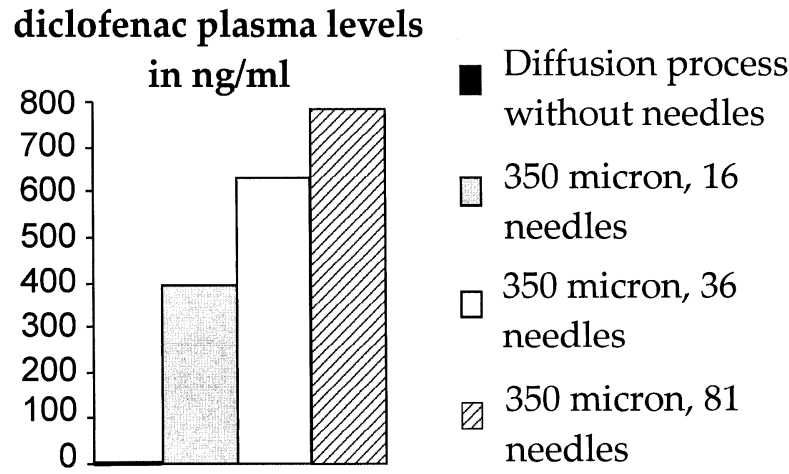

Fig. 9. Drug plasma concentrations obtained after $6 \mathrm{~h}$ application of an increasing number of microneedles (preliminary results in Sprague-Dawley 400 gr. Male rat).

chip was repetitively attached with its backside to the skin surface for $10 \mathrm{~s}$ at the same skin position as where the previous measurements without the chip were done, and $50 \mathrm{~s}$ after removal of the chip the TEWL was measured (see zone B in Fig. 8). It can be seen that no change in the TEWL occurs for this situation. This was followed by a single test in which the chip was attached for 10 seconds with its front side to the skin, such that the microneedles penetrated the skin, again at the same skin position, $50 \mathrm{~s}$ after which the TEWL was measured again (see zone $\mathrm{C}$ in Fig. 8), followed by repeat of measurements alike those in zone A (see zone D in Fig. 8). It follows from Fig. 8 that when the needles penetrate the skin the TEWL increases with a factor of 2.3, it is observed that after removal of the microneedles the skin recovers and the TEWL goes back to normal within a few minutes.

Preliminary drug infusion tests were performed by measuring the diclofenac plasma levels in a test animal after treatment with the microneedle device. The common administration route of diclofenac is oral, however, this type of administration has disadvantages, such as fast first-pass metabolism and negative side effects [29]. Alternative routes of diclofenac delivery have been proposed, and in this regard the skin has become increasingly important. Since diclofenac is not extensively absorbed through the skin due to its hydrophilic nature [30], it is expected that for this particular drug a microneedle approach will be beneficial.

The test was done with a microneedle chip mounted in a patch-like prototype (NanoPass Ltd., Israel) that was attached to the skin of the animal by an adhesive layer. Fig. 9 compares the infusion process using different microneedle arrays with the direct diffusion through the skin. It can be seen that drug infusion increases with the number of microneedles on the chip.

A more comprehensive test was performed with insulin that was transdermally delivered via an insulin pump at a rate of $1 \mathrm{U}$ (U stands for the internationally accepted insulin unit of $0.045 \mathrm{mg}$ of the pure crystalline insulin product) per hour to a small number of rats with diabetes, with a similar microneedle patch prototype as used for the diclofenac. Fig. 10 shows the change in glucose level in percentage of the baseline for different treatment techniques, where "inverted chip" means that the chip was turned with its back to the skin, resulting in transport through the same effective flow area but without mechanical assistance of the needles.

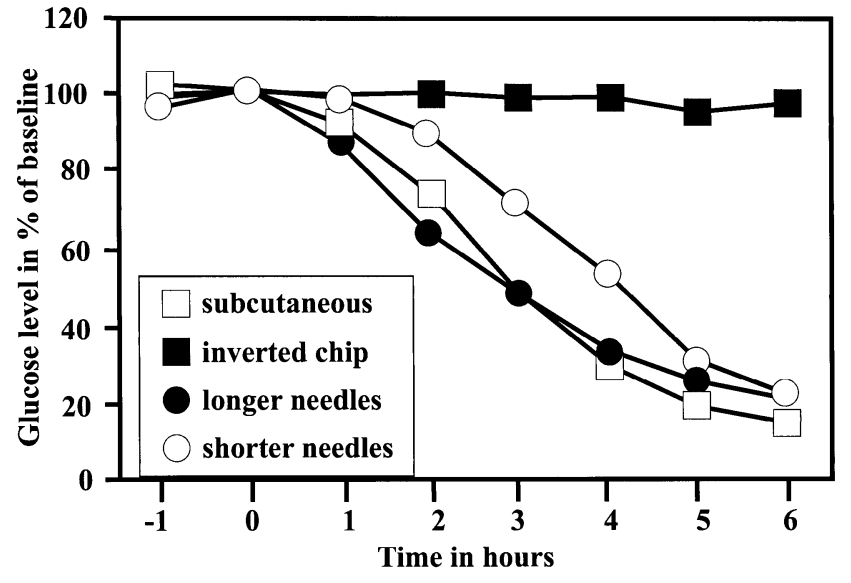

Fig. 10. Effect of insulin delivery on basal glucose levels in diabetic rats (preliminary results in small numbers of Sprague-Dawley 400 gr. Male rats); regular Insulin (Humulin R, Ely Lilly) was delivered via insulin pumps at $1 \mathrm{U} / \mathrm{h}$ through microneedle arrays, and compared to conventional subcutaneous delivery. The longer needles are $350 \mu \mathrm{m}$, the shorter ones $150 \mu \mathrm{m}$ long.

A last performance test consisted in the use of the silicon microneedle array as a diagnostic device. Since the microneedles as fabricated in this study were not long enough for transdermal blood withdrawal, the test was performed with blood collected after puncturing a human finger with a Haemolance (Haemedic $\mathrm{AB}$, Sweden) disposable safety lancet, which has a puncture depth of $1.8 \mathrm{~mm}$.

Fig. 11(a) shows a schematic of the setup as used for interfacing the microneedle array to a glass Capillary Electrophoresis (CE) chip of $2 \mathrm{~cm}$ separation length with a channel cross section of $60 \mu \mathrm{m} \times 6 \mu \mathrm{m}$ with end-column integrated electrodes for conductivity detection. The silicon needle chip was clamped onto the CE sample inlet via a PDMS seal.

In a first run, a calibrated solution of alkali ions containing $10 \mathrm{mM} \mathrm{K}{ }^{+}, \mathrm{Na}^{+}$, and $\mathrm{Li}^{+}$was electrokinetically loaded through the microneedles into the chip. A standard pinching procedure was applied to define and inject a sample plug, which was subsequently separated by Capillary Zone Electrophoresis (CZE). 20 mM 2-(N-morpholino)ethanesulfonic acid (MES; Sigma, Steinheim Germany) plus $20 \mathrm{mM}$ histidine (His; Fluka, Buchs Switzerland) was used as the background electrolyte. (BGE) Details on the CZE procedure were described earlier [31]. A reference experiment was performed with the same solutions, without the microneedle chip. Fig. 11(b) shows the resulting electropherograms. In both experiments the concentration (peak area) and migration time of the alkali ions are nearly the same, indicating that no dilution occurs after transport through the needles and confirming that the needle array does not constitute a restriction to ionic transport.

In a second experiment, ca. $30 \mu \mathrm{l}$ of the collected blood was dispensed on the microneedle chip just before the separation experiment, which was run with an identical voltage scheme. Fig. 11(c) compares the calibration graph for sampling through the needles of Fig. 11(b) with a measurement of the ions in blood. For the blood sample, two peaks were detected and identified as potassium and sodium. The ratio of concentrations of $\mathrm{K}^{+}$and $\mathrm{Na}^{+}$in whole blood, which is ca. 4:140 in blood plasma and serum, here is found to be approximately 1:1, 


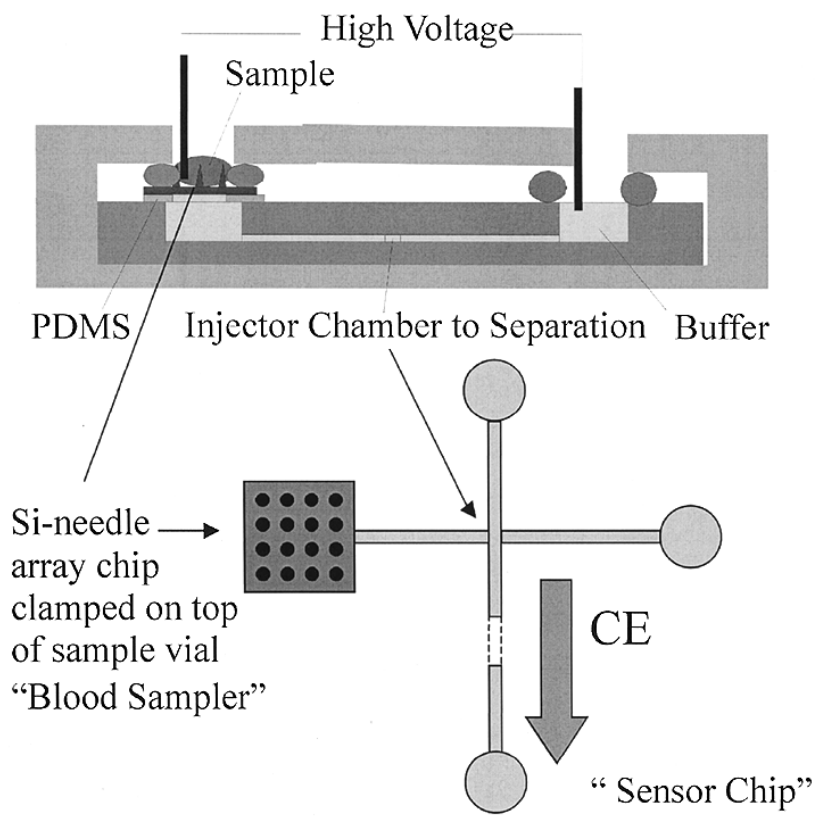

(a)

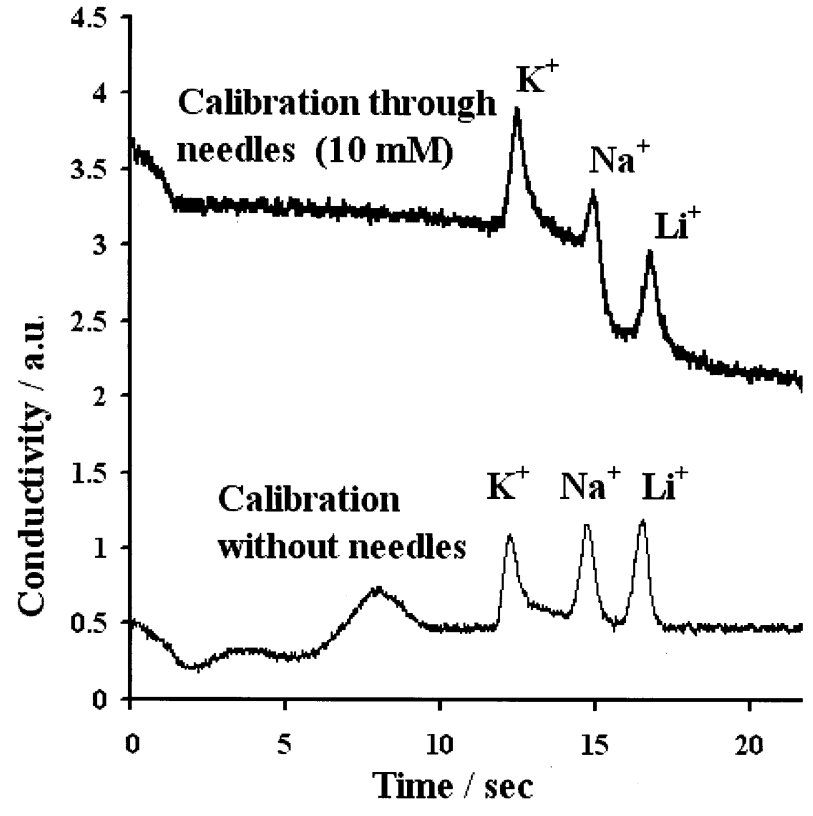

(b)

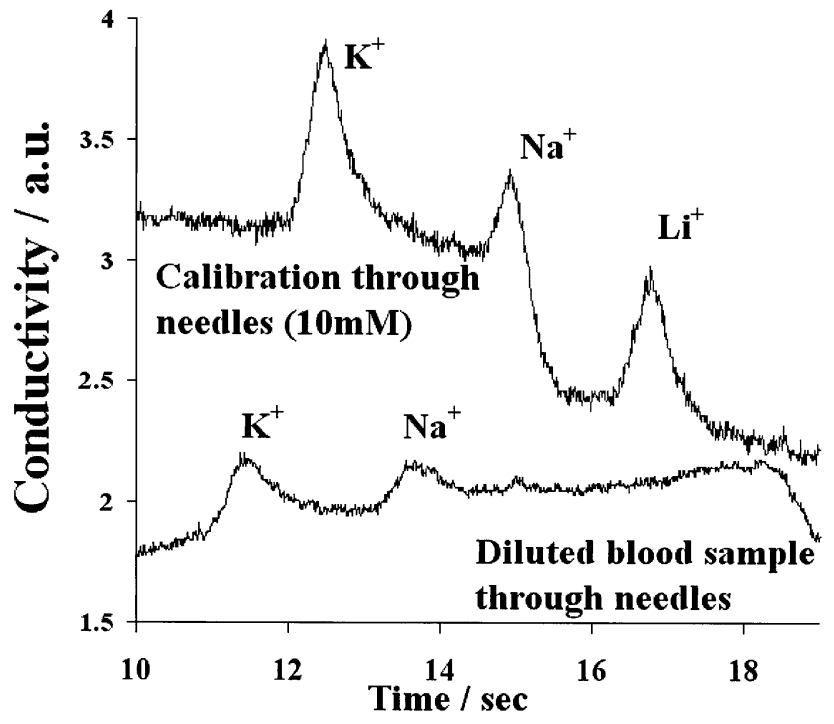

(c)

Fig. 11. (a) Measurement setup for silicon microneedle array coupled to a capillary electrophoresis chip. (b) Comparison of electrophoretic separation of calibration sample injected through the microneedles and same sample injected without the microneedle array. (c) Comparison of electrophoretic separation of whole blood sample and calibration solution both injected through the microneedle array. In all graphs of (b) and (c) sample injection occurred at time 0 s.

which is thought to be due to cell lysis during sample preparation. Furthermore, the concentration of both ions is lower than expected, indicating that dilution occurred during sample loading, which is most likely due to a phenomenon called destacking [32].

Although more experimental work needs to be done to optimize the microneedles and the blood measurement procedure, the latter experiment demonstrates the compatibility of a microneedle array sampling strategy with conventional analytical methods in a miniaturized version. This is an important step toward future portable systems for point-of-care treatments.

\section{CONCLUSION}

A new design and fabrication process for hollow microneedles in silicon was presented. The fabrication method, consisting of a sequence of DRIE, anisotropic wet etching and conformal thin film deposition steps, allows a large variety of needle shapes. Preliminary tests have shown that the microneedles have the proper mechanical strength and sharpness to be applied for transdermal applications. Several animal tests were performed, in order to evaluate the suggested medical application fields. Although no extensive clinical study was 
carried out, these tests demonstrated the feasibility of the microneedle approach. Finally, a microneedle array was used as a blood sampler on a capillary electrophoresis chip. The results for a calibrated test solution demonstrated the feasibility of this approach.

\section{ACKNOWLEDGMENT}

The authors thank B. Otter of $\mathrm{MESA}^{+}$, University of Twente, for his SEM work, and J. Bouwstra of LACDR Leiden, for stimulating discussions on skin properties and drug infusion.

\section{REFERENCES}

[1] S. Kaushik, A. H. Hord, D. D. Denson, D. V. McAllister, S. Smitra, M. G. Allen, and M. Prausnitz, "Lack of pain associated with microfabricated microneedles," Anesth. Analg., vol. 92, pp. 502-504, 2001.

[2] J. K. Chen and K. D. Wise, "A multichannel neural probe for selective chemical delivery at the cellular level," in Proc. Digest Solid-State Sensor and Actuator Workshop, Hilton Head, SC, June 1994, pp. 256-259.

[3] J. K. Chen, K. D. Wise, J. F. Hetke, and S. C. Bledsoe, "A multichannel neural probe for selective chemical delivery at the cellular level," IEEE Trans. Biomed. Eng., vol. 44, pp. 760-769, 1997.

[4] L. Lin, A. P. Pisano, and R. S. Muller, "Silicon processed microneedles," in Proc. 7th Int. Conf. Solid-State Sensors and Actuators (Transducers'93), 1993, pp. 237-240.

[5] L. Lin and A. P. Pisano, "Silicon processed microneedles," J. Microelectromech. Syst., vol. 8, pp. 78-84, 1999.

[6] K. S. Lebouitz and A. P. Pisano, "Microneedles and microlancets fabricated using SOI wafers and isotropic etching," in Proc. Microstructures and Microfabrication Systems IV, Electrochem. Soc. Proc., vol. 98-14, 1998, pp. 235-244.

[7] J. D. Brazzle, I. Papautsky, and A. B. Frazier, "Micromachined needle arrays for drug delivery or fluid extraction," IEEE Eng. Med. Biol., vol. 18 , pp. 53-58, 1999.

[8] - "Hollow metallic micromachined needle arrays," Biomed. Microdev., vol. 2, pp. 197-205, 2000.

[9] J. D. Zahn, N. H. Talbot, D. Liepmann, and A. P. Pisano, "Microfabricated polysilicon microneedles for minimally invasive biomedical devices," Biomed. Microdev., vol. 2, pp. 295-303, 2000.

[10] J. D. Zahn, D. Trebotich, and D. Liepmann, "Microfabricated microdialysis microneedles for continuous medical monitoring," in Proc. 1st Ann. Int. IEEE-EMBS Special Topic Conf. Microtechnol. in Medicine and Biol., Lyon, France, Oct. 12-14, 2000, pp. 375-380.

[11] A. C. Hoogerwerf and K. D. Wise, "A three-dimensional neural recording array," in Digest IEEE Int. Conf. on Solid-State Sensors and Actuators, San Francisco, 1991, pp. 120-123.

[12] S. Henry, D. V. McAllister, M. Allen, and M. Prausnitz, "Micromachined needles for the transdermal delivery of drugs," in Proc. IEEE Workshop MEMS, 1998, pp. 494-498.

[13] K. Chun, G. Hashiguchi, H. Toshiyoshi, H. Fujita, Y. Kikuchi, J. Ishikawa, Y. Murakami, and E. Tamiya, "An array of hollow micro-capillaries for the controlled injection of genetic materials into animal/plant cells," in Proc. IEEE Conf. MEMS, 1999, pp. 406-411.

[14] K. Oka, S. Aoyagi, Y. Isono, G. Hahiguchi, and H. Fujita, "Fabrication of microneedle for a trace blood test," in Proc. Transducers'01, Munich, Germany, June 10-14, 2001, pp. 412-415.

[15] B. Stoeber and D. Liepmann, "Two dimensional arrays of out-of-plane needles," in Proc. ASME MEMS Div., 2000 IMECE, vol. 1, 2000, pp. 355-359.

[16] P. Griss and G. Stemme, "Side-opened out-of-plane microneedles for microfluidic transdermal liquid transfer," J. Microelectromech. Syst., vol. 12, pp. 296-301, 2003.

[17] J. G. E. Gardeniers, E. J. W. Berenschot, M. J. de Boer, Y. Yeshurun, M. Hefetz, R. van't Oever, and A. van den Berg, "Silicon micromachined hollow microneedles for trandermal liquid transfer," in Proc. 15th IEEE Int. Conf. MEMS, pp. 141-144.

[18] "Microneedle Structure and Production Method Therefor," Patent application WO 0217985A2.
[19] M. J. de Boer, R. W. Tjerkstra, J. W. Berenschot, H. V. Jansen, G. J. Burger, J. G. E. Gardeniers, M. Elwenspoek, and A. van den Berg, "Micromachining of buried microchannels in silicon," J. Microelectromech. Syst., vol. 9, pp. 94-103, 2000.

[20] T. R. Albrecht, S. Akamine, T. E. Carver, and C. F. Quate, "Microfabrication of styli for the atomic force microscope," J.Vac. Sci Technol., vol. A8, pp. 3386-3396, 1990.

[21] M. J. de Boer, J. G. E. Gardeniers, H. V. Jansen, E. Smulders, M. J. Gilde, G. Roelofs, J. N. Sasserath, and M. Elwenspoek, "Guidelines for etching silicon MEMS structures using fluorine high-density plasmas at cryogenic temperatures," J. Microelectromech. Syst., vol. 11, pp. 385-401, 2002.

[22] J. G. E. Gardeniers, H. A. C. Tilmans, and C. C. G. Visser, "LPCVD silicon-rich silicon nitride films for applications in micromechanics, studied with statistical experimental design," J. Vac. Sci. Technol., vol. A 14, pp. 2879-2892, 1996.

[23] A. J. Nijdam, J. G. E. Gardeniers, J. W. Berenschot, E. van Veenendaal, J. van Suchtelen, and M. Elwenspoek, "Influence of the angle between etched (near) $\operatorname{Si}\{111\}$ surfaces and the substrate orientation on the underetch rate during anisotropic wet-chemical etching of silicon," J. Micromech. Microeng., vol. 11, pp. 499-503, 2001.

[24] A. J. Nijdam, E. van Veenendaal, H. M. Cuppen, J. van Suchtelen, M. L. Reed, J. G. E. Gardeniers, W. J. P. van Enckevort, E. Vlieg, and M. Elwenspoek, "Formation and stabilization of pyramidal etching hillocks on silicon $\{100\}$ in anisotropic etchants: experimental observations and monte carlo simulations," J. Appl. Phys., vol. 89, pp. 4113-4123, 2001.

[25] E. van Veenendaal, H. M. Cuppen, W. J. P. van Enckevort, J. van Suchtelen, A. J. Nijdam, M. Elwenspoek, and E. Vlieg, "A monte carlo study of etching in the presence of a mask junction," J. Micromech. Microeng., vol. 11, pp. 409-415, 2001.

[26] G. L. Grove, M. J. Grove, C. Zerweck, and E. Pierce, "Comparative metrology of the evaporimeter and the dermalab ${ }^{\circledR}$ TEWL probe," Skin Res. Technol., vol. 5, pp. 1-8, 1999.

[27] G. E. Nilsson, "Measurement of water exchange through skin," Med. Biol. Eng. Comp., vol. 15, pp. 209-218, 1977.

[28] G. L. Grove, M. J. Grove, C. Zerweck, and E. Pierce, "Computerized evaporimetry using the dermalab ${ }^{\circledR}$ TEWL probe," Skin Res. Technol., vol. 5, pp. 9-13, 1999.

[29] X. Hui, P. G. Hewitt, N. Poblete, H. I. Maibach, J. S. Shainhouse, and R. C. Wester, "In vivo bioavailability and metabolism of topical diclofenac lotion in human volunteers," Pharm. Res., vol. 15, pp. 1589-1595, 1998.

[30] T. Nishihata, M. Kotera, Y. Nakano, and M. Yamamazaki, "Rat percutaneous transport of diclofenac and influence of hydrogenated soya lecitin," Chem. Pharm. Bull., vol. 35, pp. 3807-3812, 1987.

[31] E. X. Vrouwe, R. Luttge, and A. van den Berg, "Measuring lithium in whole blood using capillary electrophoresis," in Proc. $\mu$ TAS 2002 Symp., Y. Baba, S. Shoji, and A. van den Berg, Eds., Nara, Japan, Nov. 3-7, 2002, pp. 178-180.

[32] E. X. Vrouwe and A. van den Berg, "Destacking loading conditions on a CE chip for measuring samples with a high matrix concentration," in 7th Int. Conf. Miniaturized Chemical and BioChemical Analysis Systems, Squaw Valley, CA, Oct. 5-9, 2003, pp. 89-92.

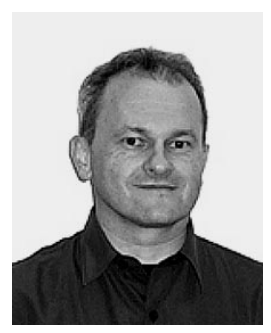

Han J. G. E. Gardeniers received the M.Sc. degree in chemistry and the Ph.D. degree in solid-state physics from the University of Nijmegen, The Netherlands, in 1985 and 1990, respectively.

In 1990, he joined the Department of Electrical Engineering at the University of Twente, The Netherlands, as an Assistant Professor. After a short period as Project Leader MEMS at Kymata Netherlands B.V. (currently: C2V B.V.) in 2001, he joined Micronit Microfluidics B.V. in September 2001, where he was responsible for R\&D related to miniaturized chemical synthesis and analysis systems. Since January 1, 2003, he has been an Associate Professor with the Department of Electrical Engineering, Mathematics and Informatics at the University of Twente, The Netherlands, within the Biosensors/Lab-on-a-Chip group. He has published over 50 reviewed journal papers and holds five patents on various topics in materials science, microfabrication and microfluidics. 


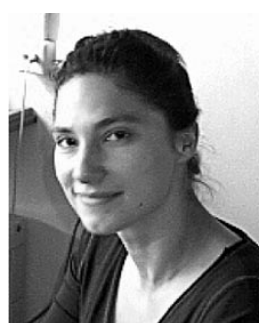

Regina Luttge received the B.Sc. degree in applied science from the Fachhochschule Wiesbaden, Germany in 1993 .

Thereafter, she worked on LIGA developments at the Institut für Mikrotechnologie Mainz (IMM), Germany, for four and one half years. After her $\mathrm{Ph} . \mathrm{D}$. project concerning microtechnological enhancements of optical scanning devices at Imperial College London, U.K., she started working as a Senior Scientist at the University of Twente, within the Biosensors/Lab-on-a-Chip group, in May 2001. Her main research expertise is in micromachining and device integration, with applications in the field of miniaturised optical, fluidic and medical analysis systems, and a recent focus on blood analysis and drug delivery. She was one of the organizers of the World Micromachine Summit in 2002

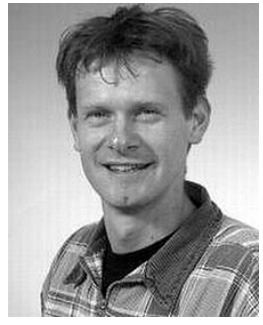

Erwin J. W. Berenschot received the B.Sc. degree in applied physics from the Technische Hogeschool in Enschede, The Netherlands, in 1990.

Since 1992, he has been employed as a micromachining engineer at the Transducer Technology group of the MESA + Research Institute. His main research area is development and characterization of etching and deposition techniques for the fabrication of micro systems. He has published over 30 reviewed journal papers on micromachining and related topics, and four patent applications.

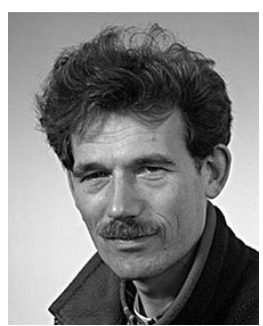

Meint J. de Boer joined the Company Sentron in Rohde, The Netherlands, as a Process Engineer in 1982, where he worked in the field of $\mathrm{pH}$-sensors and pressure sensors for medical applications. In 1988, he joined the Department of Applied Physics at the University of Groningen, The Netherlands, where he focused on nano-engineering for fundamental research on superconductivity. In 1992, he joined the Transducer Science Technology Group at the University of Twente, The Netherlands. His current research interests include silicon micromachining and related fabrication techniques, with a focus on deep reactive ion etching techniques. He has published over 20 reviewed journal papers on micromachining and related topics, and three patent applications.

Shuki Y. Yeshurun is a Senior Scientist at RAFAEL, and is the founder of NanoPass Technologies Ltd., Haifa, Israel, where he is responsible for microelectromechanical systems (MEMS) materials activities.
Meir Hefetz formerly was leader of the special technologies group at the RAFAEL Advanced Materials and Processes Department, and is now Senior Scientist at NanoPass Technologies Ltd., Haifa, Israel.

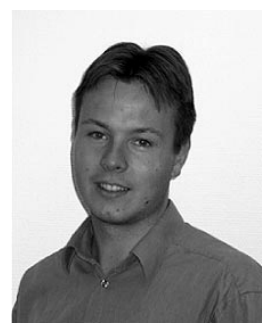

Ronny van't Oever received the M.Sc. degree in applied physics from the University of Twente, The Netherlands, in 1999. He graduated on the development of the world's smallest micropipette, fabricated by silicon micromachining, and to be used for clamping single DNA fragments.

After his graduation, he founded the company "Micronit Microfluidics B.V.," in which he presently holds the CTO position.

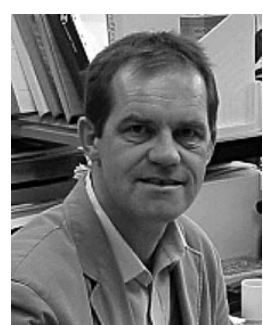

Albert van den Berg received the M.Sc. and Ph.D. degrees in applied physics from the University of Twente, The Netherlands, in 1983 and 1988, respectively.

From 1988 to 1990, he was at the Swiss Center for Microelectronics and Microtechnology (CSEM) in Neuchâtel, Switzerland, as a Project Manager in the chemical sensors department. From 1990 to 1993, he investigated miniaturized chemical sensor systems at the Institute of Microtechnology (IMT) at the University of Neuchâtel. In 1993, he began, and since then, has led the strategic research orientation "Micro (Bio) Chemical Systems" (MiCS) at the MESA + Research Institute at the University of Twente, where he was appointed full professor in 1998. He holds the chair "Biosensors/Lab-on-aChip" which is part of the Department of Electrical Engineering, Mathematics and Informatics at the University of Twente, The Netherlands, since August 2002. He has published more than 70 reviewed papers on $\mu$ TAS-related subjects. His current research interests are theory, technologies, new devices and applications of micro- and nanofluidics for miniaturized (bio) chemical synthesis, analysis and dosing systems.

Dr. van den Berg is a member of the scientific committee of the European Conference on Micro- and Nanotechnologies for Life Sciences (NanoTech), the scientific committee of the $\mu$ TAS conferences, the $\mu$ TAS steering committee, the advisory board of the 4th Int. Micro-machine Symposium, the steering committee of the International Transducers Conference series, and is chair of the Gordon Research Conference on Physics and Chemistry of Micro Fluidics in 2003 and organizer of the Micromachine Summit in 2002. In 2002, he received the prestigious Simon Stevin Meester award from STW, the Dutch Technology Foundation. 